\title{
MicroRNA-26b inhibits cardiac remodeling after myocardial infarction by targeting ring finger protein 6 expression
}

\author{
Chun-Mei Tang ${ }^{1,2}$, Qiang Su ${ }^{1,2}$, Hai-Xia Zhao ${ }^{1,2}$, He-Huan Sui ${ }^{1,2}$, Jing Liang ${ }^{1,2}$, Li-Sha Zhu ${ }^{1,2}$, \\ Si-Yun Yang ${ }^{1,2}$, Tao Liu'
}

\begin{abstract}
1Department of Pharmacy, Nanchong Central Hospital, The Second Clinical Medical College, North Sichuan Medical College (University), Nanchong, Sichuan, China ${ }^{2}$ Nanchong Key Laboratory of Individualized Drug Therapy, Nanchong, Sichuan, China ${ }^{3}$ Cardiovascular Medicine, Nanchong Central Hospital, The Second Clinical Medical College, North Sichuan Medical College (University), Nanchong, Sichuan, China
\end{abstract}

Submitted: 26 May 2020; Accepted: 16 November 2020

Online publication: 17 April 2021

Arch Med Sci

DOI: https://doi.org/10.5114/aoms/130649

Copyright $\odot 2022$ Termedia \& Banach

\section{Abstract}

Introduction: This study aimed to determine the regulatory mechanism of miR-26b in myocardial infarction (MI)-induced cardiac remodeling through apoptosis.

Material and methods: An MI rat model was established by left coronary artery ligation. Microarray data were analyzed to distinguish differentially expressed genes in MI. miR-26b was found to be poorly expressed, whereas ring finger protein 6 (RNF6) was highly expressed in MI. Consequently, miR-26b was identified to target RNF6 using dual-luciferase reporter assay and bioinformatics prediction. Furthermore, rats injected with a lentiviral vector expressing miR-26b mimic and/or RNF6 were used to evaluate the role of miR-26b and RNF6 in regulating cardiac function, infarct size, and cardiomyocyte apoptosis.

Results: miR-26b overexpression improved cardiac function and increased left ventricular end-diastolic and end-systolic diameters. Meanwhile, increased miR-26b expression decreased infarct size and cardiomyocyte apoptosis. Moreover, RNF6 overexpression counteracted the role of miR-26b in cardiac function. Additionally, an in vitro cell model illustrated that miR-26b upregulation could increase cell viability and reduce apoptosis, whereas RNF6 overexpression reversed its effect. We also found that the miR-26b mimic could negatively modulate RNF6 expression to inactivate the ER $\alpha$ / Bcl-xL axis.

Conclusions: miR-26b plays a protective role against cardiac remodeling after MI through inactivation of the RNF6/ER $\alpha / B C l-x L$ axis, supporting miR-26b and RNF6 as potential therapeutic targets for MI.

Key words: myocardial infarction, cardiac remodeling, microRNA-26b, ring finger protein 6 , cardiac function, rats

\section{Introduction}

Myocardial infarction (MI) is the prevalent sign of adverse left ventricular (LV) remodeling and heart failure (HF), and a major trigger of morbidity and death worldwide [1, 2]. Despite the combined use of pharmacological and emergency reperfusion treatments, adverse LV remodeling

\author{
Corresponding author: \\ Tao Liu \\ Cardiovascular Medicine \\ Nanchong Central Hospital, \\ The Second Clinical \\ Medical College \\ North Sichuan Medical \\ College (University) \\ No. 96 Dabei Street, \\ Shunqing District \\ Nanchong 637000, \\ Sichuan, China \\ Phone: 0817-2258096 \\ E-mail: taoliu27@163.com
}


remains associated with unfavorable outcomes $[2,3]$. Hence, the development of new treatment schemes able to effectively suppress or reverse adverse postischemia LV remodeling and dysfunction is needed. MicroRNAs (miRs), which are small noncoding RNAs, can modulate gene expressions following transcription [4], and it is estimated that most genes and cell processes are regulated by molecules such as miRs $[5,6]$. miRs are also related to various diseases [7], including cardiovascular diseases [8]. Cardiac remodeling (CR) is the most interesting phenomenon in such conditions. Distinct miRs that play a vital role in cardiomyocyte hypertrophy include miR-208, -133 , and $-212 / 132$ [9-11], whereas miR-21, -30, -133, and, -29 have been confirmed to be involved in fibrosis [12-16]. In this aspect, the relevance of miR-26a/b in some cardiac diseases has been established [17-23]. Taken together, these observations confirmed the hypothesis of miR-26b being a potential target for ameliorating cardiac function. Remarkably, a study demonstrated that miR-26a suppresses breast cancer cell growth by inhibiting RNF6/ER / $\mathrm{BCl}-\mathrm{xL}$ signaling [24]. Accordingly, we hypothesized that miR-26b is involved in CR after MI. The present study was conducted to verify this hypothesis based on an MI rat model that was established by left coronary artery ligation and to assess the potential regulatory mechanism associated with

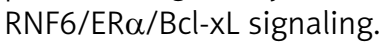

\section{Material and methods}

\section{Animals}

Animal experiments were approved by the Institutional Animal Care and Use Committee of the North Sichuan Medical College (No. 064/11 and R13-5424). All in vivo experiments were conducted using male adult Wistar rats (weight: 250-300 g).

\section{Myocardial infarction rat model and miR profiling}

In rats, $\mathrm{MI}$ was induced by ligating the left anterior descending artery, as previously described $[25,26]$. On day 14 after $\mathrm{Ml}$ induction, cardiac tissues were obtained for miR microarray. MiR expression was profiled using concentrated samples of six rats from each group at all time points on day 14 after MI induction, as previously described [27].

\section{Construction of a lentivirus vector}

HEK293T cells in the logarithmic phase were employed for lentivirus packaging. After trypsinization and counting, cells $\left(5 \times 10^{5}\right.$ cells $\left./ \mathrm{ml}\right)$ were inoculated into a $10-\mathrm{cm}^{2}$ culture dish. The complete medium was replaced with serum-free medium $2 \mathrm{~h}$ before cell infection. When cell confluence reached approximately $80 \%$, cells were infected with Lipofectamine 2000. Next, $2.5 \mathrm{ml}$ of solution A (Opti-MEM supplemented with $25 \mu \mathrm{g}$ of GV320 lentiviral vector expressing miR-26b mimic, miR-NC, RNF6, or empty vector; $12.5 \mu \mathrm{g}$ of pHelper1.0; and $10 \mu \mathrm{g}$ of pHelper2.0) and solution B $(10 \mu \mathrm{l}$ of Lipofectamine 2000 and $2.5 \mathrm{ml}$ of Opti-MEM) were mixed at room temperature for $20 \mathrm{~min}$. HEK293T cells were cultured with a mixture of solutions $A$ and $B$ at $37^{\circ} \mathrm{C}$ with $5 \% \mathrm{CO}_{2}$. After $8 \mathrm{~h}$ of culture, cells were completely washed with phosphate-buffered saline, removed from cultured medium, and continued to be cultured with a medium containing $10 \%$ fetal bovine serum for $48 \mathrm{~h}$. Subsequently, the supernatant of HEK239T cells was collected and centrifuged at $2000 \mathrm{~g}$ at $4^{\circ} \mathrm{C}$ for $10 \mathrm{~min}$ to remove cell debris. Cells filtered using a $0.45-\mu \mathrm{m}$ filter were collected and preserved at $-80^{\circ} \mathrm{C}$.

\section{Animal treatment}

Control rats (control group; sham-operated, punctured without coronary artery ligation) and $\mathrm{MI}$ rat models (model group) were not subjected to any delivery of lentivirus, and the two groups comprised 20 male rats each. The model group ( $n=80,50 \%$ male and $50 \%$ female) rats were injected with the lentivirus through the tail vein: miR-NC, miR-26b, combination of miR-26b and lentiviral-NC, and combination of miR-26b and RNF6; each group comprised 10 male and $10 \mathrm{fe}$ male rats. The lentivirus injection $\left(1 \times 10^{7} \mathrm{PFU}\right.$ per rat) through the tail vein was performed within $300 \mathrm{~s}$ after modeling and 3 days after modeling again. On day 13 after MI induction, cardiac function was assessed with the Vevo 770 ultrasound imaging system (Visual Sonics). Then, the rats were euthanatized, and the cardiac tissues were collected for subsequent experiments.

\section{Echocardiography}

On day 7 after $\mathrm{MI}$ induction, the rats were anesthetized with $1 \%$ isoflurane. During echocardiography, isoflurane (1.5-2.0\%) and oxygen were supplied to maintain anesthesia. Cardiac function parameters including LV end-diastolic diameter (LVEDD) and volume (LVEDV) and LV end-systolic diameter (LVESD) and volume (LVESV) were determined and recorded using ultrasound biomicroscopy InviVue (Vevo 770; Visual Sonics) with an RMV 704 probe of $40 \mathrm{MHz}$ central frequency. Thereafter, LV fractional shortening (LVFS) and ejection fraction (LVEF) were acquired [28], and the average values was determined after three successive cardiac cycles. 


\section{Tissue collection}

The rats were euthanized on day 14 after MI induction, and their hearts were quickly excised. Myocardial tissues were extracted from the apex of the heart to the ligature site along the short axis using a blade, and the tissues were sliced into 2-mm-thick sections, followed by triphenyl tetrazolium chloride (TTC) staining. Meanwhile, myocardial tissues were extracted from the infarct areas. Parts of myocardial tissues were preserved for subsequent quantitative reverse transcription polymerase chain reaction (RT-qPCR) and western blotting (WB).

\section{Cell cultivation and transfection}

H9C2 cells and HEK293T cells were purchased from ATCC and cultivated in accordance with the manufacturer's instructions. To investigate the effect of $\mathrm{H}_{2} \mathrm{O}_{2}$ treatment on miR-26b expression and relevant objective genes, cells were inoculated for 1 day, preserved in a standardized medium, and maintained under normoxic and $\mathrm{H}_{2} \mathrm{O}_{2}(100 \mu \mathrm{M})$ conditions for 2 days. Cells were subsequently collected for RT-qPCR. To evaluate the effects of RNF6 and miR-26b in vitro, cells were treated with LNA-modified miR-26b mimic, NC mimic, or a combination of miR-26b with pcDNA3-NC or pcDNA3-RNF6 at different concentrations and maintained under normoxic or $\mathrm{H}_{2} \mathrm{O}_{2}$ conditions for two days. Furthermore, cells were obtained for RT-qPCR, WB, and cell proliferation and apoptosis assays.

\section{Cell growth}

Cell viability capacity was evaluated using the CCK- 8 assay according to the manufacturer's instructions. Cells were seeded into 96-well plates, CCK-8 $(10 \mu \mathrm{l})$ was added to each well and cells were incubated for a further $2 \mathrm{~h}$ at $37^{\circ} \mathrm{C}$. Optical density was measured at $450 \mathrm{~nm}$ using an auto-microplate reader (infinite M200, Tecan, Switzerland), as mentioned previously [29, 30].

\section{Dual-luciferase reporter assay (DLRA)}

TargetScan Human 7.2 (http://www.targetscan.org/vert_72/) was applied to identify the miR-26b-5p-binding sites on RNF6 3'-UTR. After amplification, the fragments of RNF6 mRNA with possible mutants (MUT) or WT miR-26a-5p-binding sites were cloned into a pGL3 vector (50 ng), which were then cotransfected to HEK293T cells for 2 days with miR-26b-5p or miR-NC (100 ng) through Lipofectamine 2000 (Invitrogen, USA). The pRL-TK-Renilla vector (25 ng) that provided the constitutive expression of Renilla luciferase was cotransfected as an internal control to correct the differences in both transfection and harvest efficiencies. In the end, relative luciferase activity was detected by a GloMax 96 Luminometer based on the recommendations of Dual-Glo Luciferase Assay System (Promega, Madison, WI, USA). All experiments were performed in triplicate.

\section{Western blotting}

Cells were treated with the protease inhibitor cocktail (Roche Applied Science) and radioimmunoprecipitation assay buffer $(\mathrm{pH}$ 8.0). Protein concentration was measured using a bicinchoninic acid kit. Proteins were separated with sodium dodecyl sulfate polyacrylamide gel electrophoresis and electrically added to polyvinylidene difluoride membranes (Millipore, MA, USA). The available sites were blocked by incubating with primary antibodies at $4^{\circ} \mathrm{C}$ overnight and subsequently rinsed with Tris-buffered saline and tween 20 (TBST). Moreover, immunoblots were observed by cultivating cells with secondary antibodies for $60 \mathrm{~min}$ at room temperature. The bands observed on membranes were rinsed with TBST and visualized using the Thermo Maximum Sensitivity Substrate Kit (MA, USA).

\section{Reverse transcription polymerase chain reaction}

Total RNA was extracted from cells and tissue specimens (0.100 g) with TRIzol (Invitrogen), and the concentration was determined using NanoDrop2000 $\left(\mathrm{OD}_{260}\right)$. cDNA was prepared through reverse transcription using an Oligo(dT)20 primer, and the MMLV First-Strand Synthesis Kit (Invitrogen) for RT-qPCR. RT-qPCR detection of U6 and miR-1225 was performed using relevant kits, according to the manufacturer's instruction. The RT-qPCR conditions were as follows: denaturation for $10 \mathrm{~min}$ at $95^{\circ} \mathrm{C}, 40$ denaturation cycles for $15 \mathrm{~s}$ at $95^{\circ} \mathrm{C}$, and extension for $40 \mathrm{~s}$ at $60^{\circ} \mathrm{C}$. Glyceraldehyde-3-phosphate dehydrogenase or U6 mRNA expressions served as the internal control. The $2^{-\Delta \Delta C T}$ method was performed to measure the target mRNA expression levels. All procedures were conducted in parallel three times.

\section{Flow cytometry}

Cell apoptosis was assessed by flow cytometry (FCM) with an Annexin V-FITC/PI apoptosis detection kit (BD Pharmingen). Cells were suspended in a binding buffer $(0.02 \mathrm{ml})$ and exposed to Annexin V-FITC $(0.01 \mathrm{ml}) / \mathrm{PI}(5 \mu \mathrm{l})$. Cell apoptosis rates were determined through FCM.

\section{Triphenyltetrazolium chloride staining}

The myocardial infarct size was determined by triphenyltetrazolium chloride (TTC) staining. Brief- 
ly, the rat heart was sliced into small sections which were incubated with 1\% TTC (A610558, SangonBootech Co, Shanghai, China) for 30 min, fixed with $10 \%$ formalin for $10 \mathrm{~min}$, and then observed after rinsing. The infarct area (pale white) and the area at risk (brick red) were measured using Image-Pro Plus 6.0 software. The infarct size (\%) was calculated as infarct area/area at risk $\times 100 \%$.

\section{Data analysis}

All data are presented as mean \pm standard deviation (SD). ANOVA was applied for determining differences among groups and the $t$-test for determining differences between two groups. A $p$-value of $<0.05$ was considered statistically significant.

\section{Results}

\section{miR expression profiles after myocardial infarction}

To explore the miR expression profiles in cardiac impairment, the expression profiles of miRs isolated from cardiac tissues from the model and control groups (six samples per group) were determined on day 14 after MI induction. Differentially expressed genes (DEGs) were selected from the GSE46395 microarray data with an adjusted $p$-value of $<0.05$ and $|\log 2 \mathrm{FCM}|$ of $>2.0$ as the threshold, and a heatmap of the top 17 DEGs was constructed (Figure 1 A). Furthermore, miR-26b expression in myocardial tissues was determined using RT-qPCR after successful establishment of the $\mathrm{MI}$ rat model, confirming the decrease in miR$26 \mathrm{~b}$ expression level in myocardial tissues from the model group compared with those from the control group (Figure $1 \mathrm{~B}$ ).

A

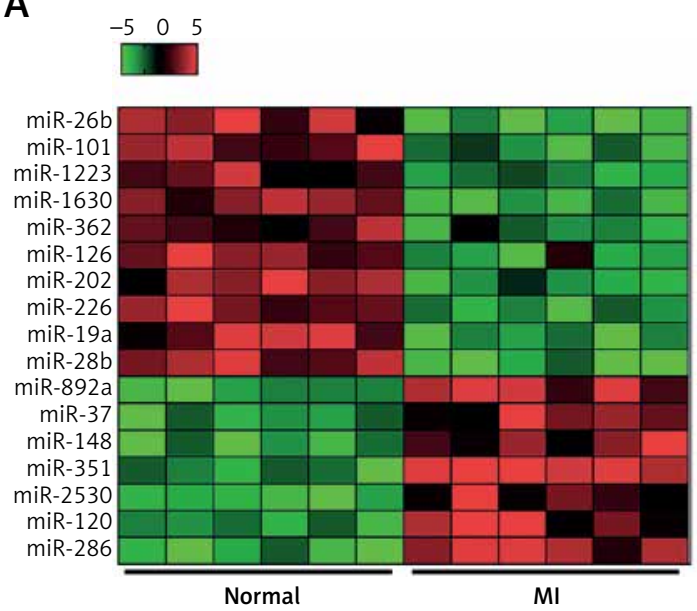

\section{MiR-26b targets RNF6 3'-UTR}

Subsequently, we performed bioinformatics prediction to identify the putative target of miR26b. The obtained data indicated that RNF6 3'UTR has binding sites on miR-26b (Figure 2 A). Dual-luciferase reporter assay data then confirmed that RNF6 was a target gene of miR-26b, and miR-26b mimic transfection can inhibit the downregulation of luciferase activity via wild-type RNF6 (Figure 2 B). To further examine the effect of miR-26b upregulation on RNF6 expression in the model group, lentiviral-miR-26b/NC was used and injected in the model group to regulate miR-26b expression. We first detected miR-26b expression in the model group. RT-qPCR showed that lentiviral-miR-26b administration resulted in elevated miR-26b expression levels compared with those in the lentiviral-miR-NC group (Figure $2 \mathrm{C}$ ). Then, RT-qPCR and WB demonstrated that Ml-induced cardiac modeling induced RNF6 expression, whereas miR-26b upregulation significantly decreased RNF6 expression in both mRNA and protein levels (Figures 2 D, E), suggesting that RNF6 expression was negatively correlated with miR$26 \mathrm{~b}$ in myocardial tissues from the model group.

\section{miR-26b improved cardiac function after $\mathrm{MI}$ by targeting RNF6}

RNF6 was a target gene of miR-101; thus, its effects on $\mathrm{MI}$ were explored in the model group injected with lentiviral-miR-26b, lentiviral-miR-NC, lentiviral-miR-26b+lentiviral-NC, or lentiviral-miR-26b+lentiviral-RNF6. Subsequently, the indicators of cardiac function (LVEDD, LVESD, LVFS, and LVEF) were measured, aiming to dis-

B

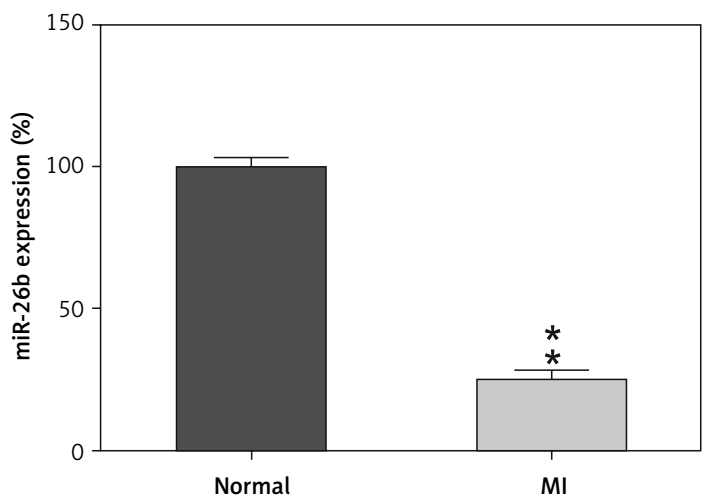

Figure 1. MicroRNA-26b (miR-26b) was a downregulated gene in cardiac tissues from rats with MI. A - The heatmap of the top 17 differentially expressed genes (DEGs) in microarray data of GSE46395. The abscissa and ordinate present sample number and DEGs, respectively, and the upper right histogram represents color grade. Each rectangle in the image represents the expression of a sample. B - miR-26b expression in myocardial tissues in the control and MI groups determined by RT-qPCR

$\mathrm{MI}-$ myocardial infarction, ${ }^{* *} p<0.01$ vs. control group only. 
A

miR-26b
RNF6 WT 'UCGgAUAGGACCUAAUGAACUU-3'
RNF6 MUT

B

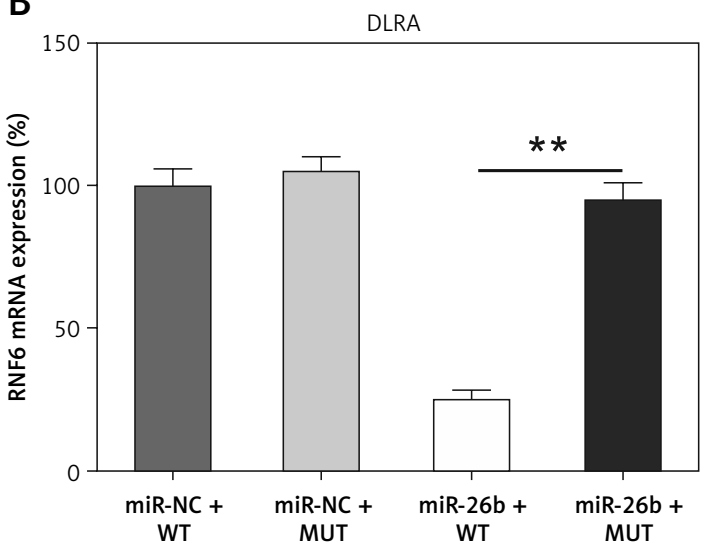

D

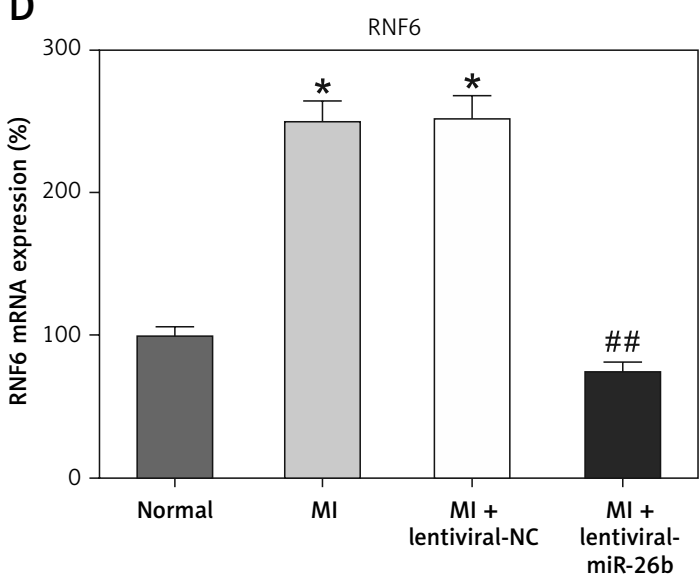

C

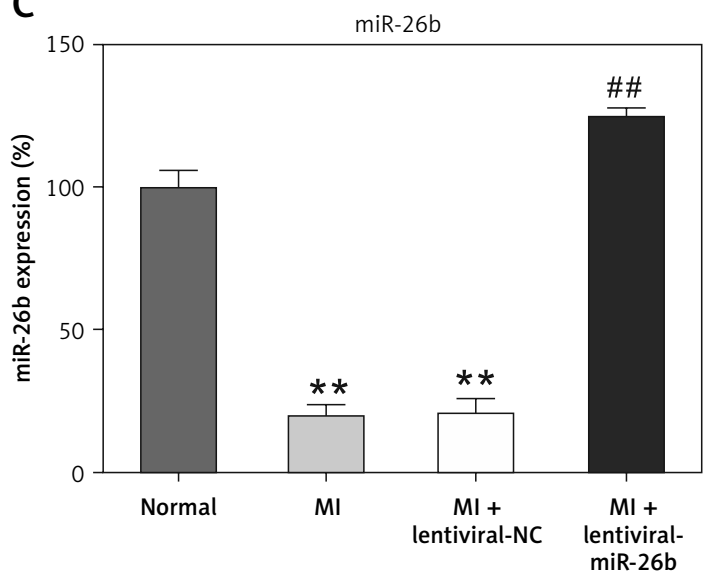

$\mathbf{E}$

RNF6

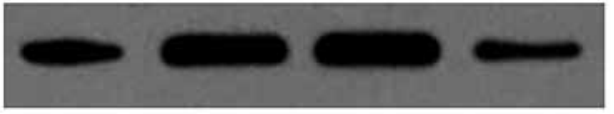

Actin

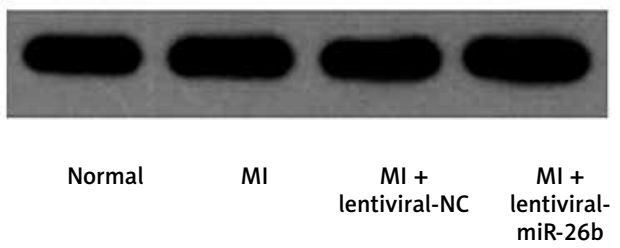

Figure 2. MicroRNA-26b (miR-26b) targets RNF63'-UTR. A - Bioinformatics analysis indicating that miR-26b possesses a binding site on RNF6 3'-UTR. B - DLRA was conducted, followed by dual-luciferase reporter assay with a wild-type or mutated RNF6 mRNA, and miR-26b or NC mimic were cotransfected into HEK293T cells. MI rats used were injected with lentiviral vectors expressing miR-NC or miR-26b. C-RT-qPCR showed miR-26b expression in myocardial tissues from MI rats. D, E-RT-qPCR and WB showed RNF6 mRNA and protein expression in myocardial tissues from $M I$ rats

${ }^{*} p<0.05,{ }^{* *} p<0.01 \mathrm{vs}$. indicated/control groups, ${ }^{\# \#} p<0.01 \mathrm{vs}$. MI groups.

cover the effects of miR-26b and RNF6 on cardiac function. Echocardiography revealed that LVEDD and LVESD were significantly higher, whereas LVFS and LVEF were markedly lower in the model group compared with the sham-operated group (Figures 3 A-D). Lentiviral-miR-26b administration led to reduced LVEDD and LVESD and elevated LVFS and LVEF in the model group. However, compared with the $M I$ rats injected with lentiviral-miR-26b, those injected with lentiviral-miR-26b + lentiviral-RNF6 displayed high LVEDD and LVESD but low LVFS and LVEF, contradicting the results of miR-26b upregulation. These data suggested that MI-induced cardiac dysfunction can be relieved with miR-101 upregulation and that RNF6 was involved in this process.

\section{miR-26b reduces myocardial infarct area in $\mathrm{MI}$ rats by targeting RNF6}

Triphenyl tetrazolium chloride staining was performed to explore the effects of miR-26b and RNF6 on myocardial infarct size in MI rats. Compared with control rats, $\mathrm{Ml}$ rats had a larger heart volume, a thinner LV wall, and an obvious white myocardial infarct area. The myocardial infarct area in $\mathrm{MI}$ rats significantly decreased after treatment with lentiviral-miR-26b mimic but significantly increased after treatment with len- 
A

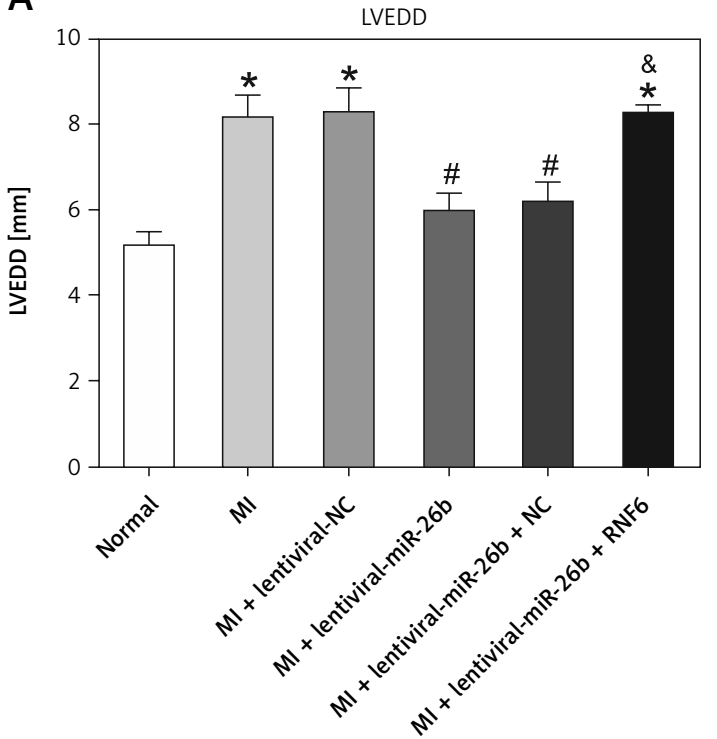

C

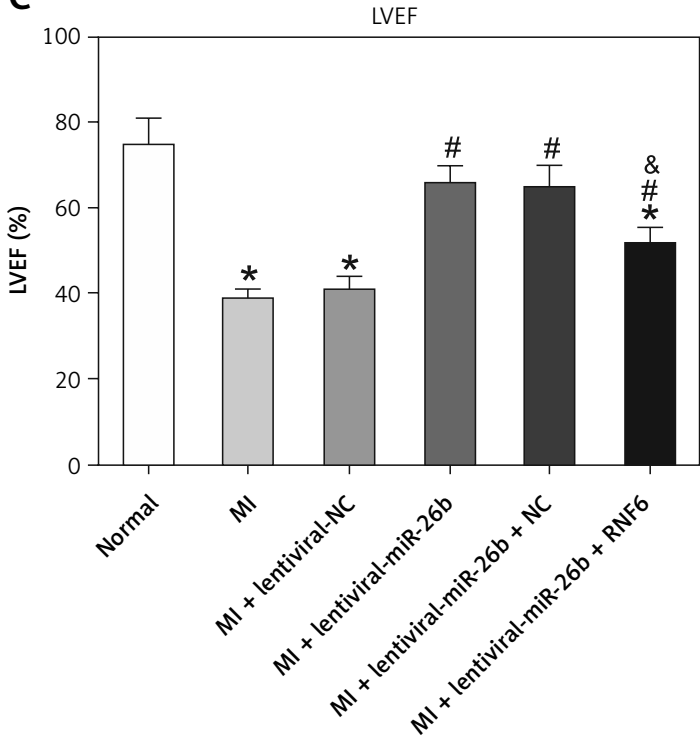

B

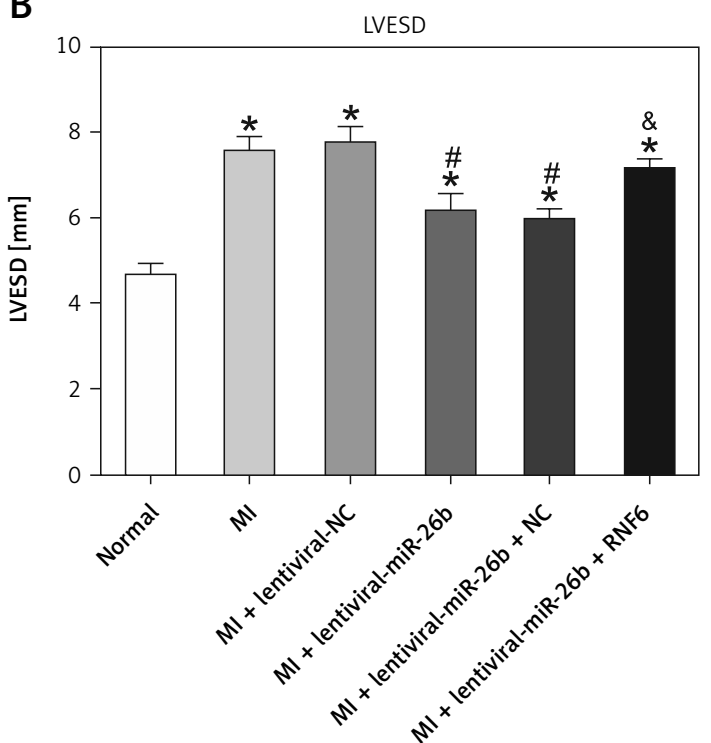

D

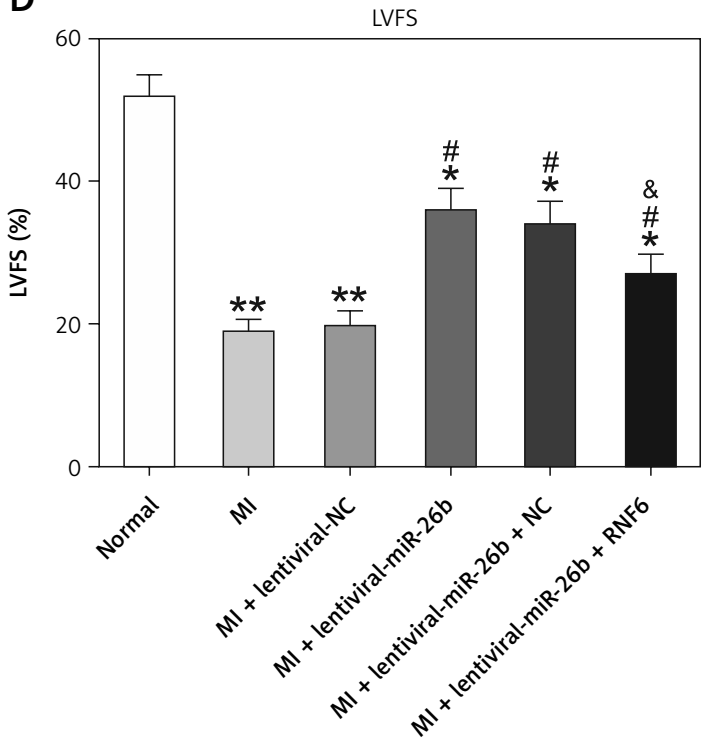

Figure 3. MicroRNA-26b (miR-26b) overexpression improved cardiac function in MI rats. The MI rats used were injected with lentiviral vectors expressing miR-NC, miR-26b, miR-26b+NC, and/or miR-26b+RNF6. A - Left ventricular end-diastolic diameter (LVEDD) in control and MI rats. B - Left ventricular end-systolic diameter (LVESD) in control and MI rats. C - Left ventricular ejection fraction (LVEF) in control and MI rats. D - Left ventricular fractional shortening (LVFS) in control and MI rats

${ }^{*} p<0.05,{ }^{* *} p<0.01$ vs. control rats; ${ }^{*} p<0.05$ vs. MI rats; ${ }^{*} p<0.05$ vs. MI rats subjected to both lentiviral vectors expressing $\operatorname{miR}-26 b$.

tiviral-miR-26b+lentiviral-RNF6 (Figures $4 \mathrm{~A}, \mathrm{~B}$ ). Taken together, the results show that miR-26b overexpression contributed to decreased myocardial infarct size in MI rats, whereas RNF6 overexpression reversed this effect.

\section{miR-26b suppresses cardiomyocyte apoptosis in MI rats by targeting RNF6}

The effects of miR-26b and RNF6 on cardiomyocyte apoptosis were determined by testing two typical apoptosis markers, i.e., Bcl-2 and Bax, in miR-26b or RNF6 restoration in cardiomyocytes. As demonstrated by RT-qPCR and WB, MI rats displayed lower Bcl-2 and higher Bax expression levels in both mRNA and protein levels than control rats (Figures $5 \mathrm{~A}-\mathrm{C}$ ). Compared with the $\mathrm{MI}$ rats injected with empty vector, the $M I$ rats with miR-26b upregulation had considerably higher $\mathrm{Bcl}-2$ and lower Bax expression levels in cardiomyocytes. Meanwhile, the $\mathrm{MI}$ rats injected with lentiviral-miR-26b and RNF6-overexpressing vector presented with markedly lower $\mathrm{Bcl}-2$ and high- 
A

Normal

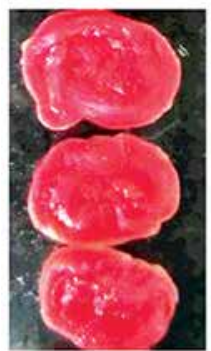

MI

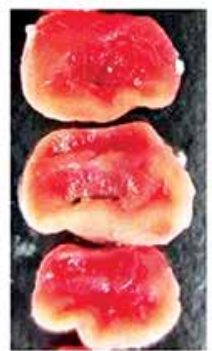

$\mathrm{MI}+$ lentiviral-NC

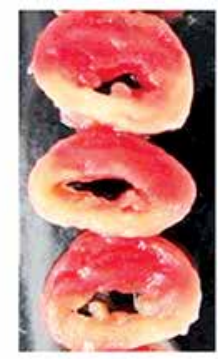

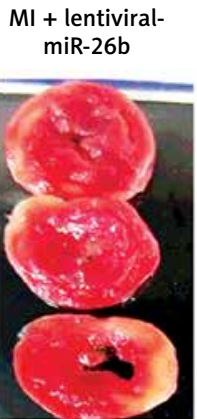

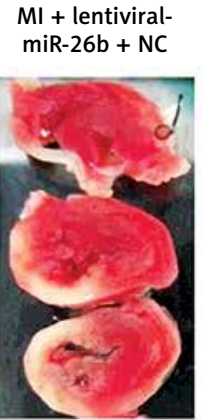

$\mathrm{MI}+$ lentiviralmiR-26b + RNF6

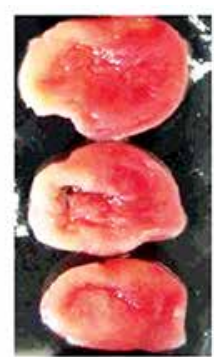

B

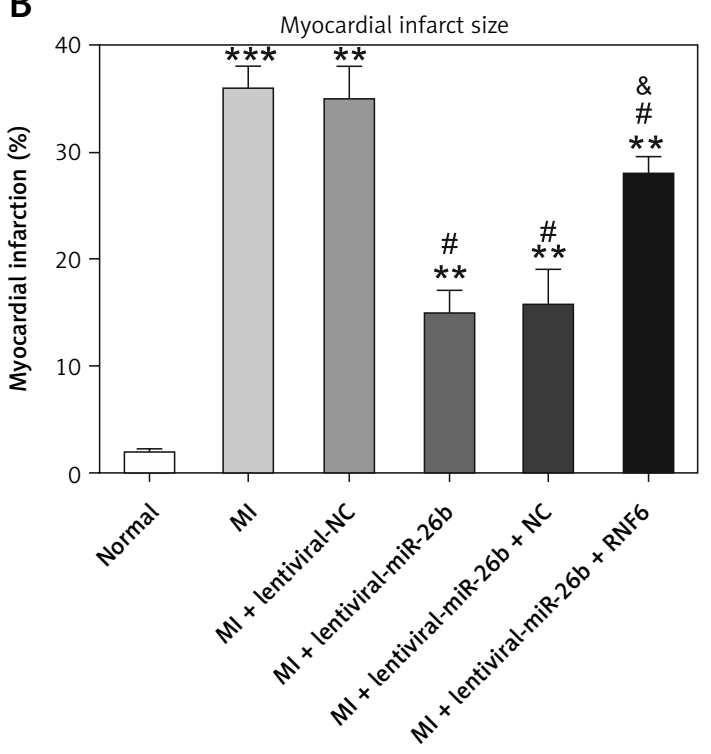

er Bax expression levels compared with $\mathrm{MI}$ rats injected with lentiviral-miR-26b (Figures $5 \mathrm{~A}-\mathrm{C}$ ). Collectively, miR-26b upregulation or RNF6 restoration could contribute to inhibited or promoted cardiomyocyte apoptosis after MI, respectively.

\section{miR-26b upregulation promoted survival} and inhibited cell apoptosis of $\mathrm{H}_{2} \mathrm{O}_{2}$-treated H9C2 cells

$\mathrm{H}_{2} \mathrm{O}_{2}$ has been widely reported to induce injury and apoptosis of cardiomyocytes, and $\mathrm{H}_{2} \mathrm{O}_{2}$-treated cardiomyocytes served as a cell model for $\mathrm{MI}$ in this study [31]. At first, RT-qPCR and WB showed that miR-26b and RNF6 were downregulated and upregulated in $\mathrm{H}_{2} \mathrm{O}_{2}$-treated $\mathrm{H} 9 \mathrm{C} 2$ cells, respectively, which is consistent with their performance in the $\mathrm{MI}$ rat model (Figures $6 \mathrm{~A}-\mathrm{C}$ ). Thereafter, $\mathrm{H}_{2} \mathrm{O}_{2}$-treated $\mathrm{H} 9 \mathrm{C} 2$ cells were transfected with miR-26b mimic and/or RNF6-overexpressing vector to induce miR-26b overexpression and RNF6 restoration. Transfection with miR-26b mimic obviously led to an increased miR-26b level in cells (Figure $6 \mathrm{~A}$ ). Meanwhile, cotransfection of miR-26b mimic and pcDNA3-RNF6 also caused
Figure 4. Myocardial infarct size in $\mathrm{MI}$ rats was reduced in response to elevation of microRNA-26b (miR-26b) expression level. MI rats used were injected with lentiviral vectors expressing miR-NC, miR-26b, miR-26b+NC, and/or miR-26b+RNF6. $A$ - Representative images of myocardial infarct in control and $\mathrm{MI}$ rats detected by triphenyl tetrazolium chloride staining. B - Percentage of myocardial infarct area in control and $\mathrm{MI}$ rats

${ }^{* *} p<0.01,{ }^{* * *} p<0.001$ vs. control rats. ${ }^{*} p<0.05$ vs. MI rats. ${ }^{\&} p<0.05$ vs. Ml rats injected with lentiviral vector expressing miR-26b.

an increase in RNF6 upregulation in $\mathrm{H}_{2} \mathrm{O}_{2}$-treated $\mathrm{H} 9 \mathrm{C} 2$ cells with a high miR-26b expression level (Figures 6 B, C). Furthermore, WB and RT-qPCR were performed to assess $\mathrm{BCl}-2$ and Bax expression levels. $\mathrm{H}_{2} \mathrm{O}_{2}$ treatment downregulated $\mathrm{BCl}-2$ expression but upregulated Bax expression. In contrast, transfection with miR-26b mimic resulted in a considerably lower Bax expression level compared with transfection with NC mimic. miR26b overexpression also promoted $\mathrm{Bcl}-2$ expression (Figures $6 \mathrm{C}-\mathrm{E}$ ). However, RNF6 restoration contributed to an elevated Bax level and attenuated $\mathrm{Bcl}$-2 level in $\mathrm{H}_{2} \mathrm{O}_{2}$-treated $\mathrm{H} 9 \mathrm{C} 2$ cells with miR26b upregulation (Figures $6 \mathrm{C}-\mathrm{E}$ ). Flow cytometry was also performed to test the apoptosis rate of $\mathrm{H} 9 \mathrm{C} 2$ cells subjected to different treatments. $\mathrm{H}_{2} \mathrm{O}_{2}$ administration caused significant death of $\mathrm{H}_{2} \mathrm{CC}_{2}$ cells, whereas miR-26b upregulation significantly reduced the proportion of apoptotic cells to a normal level. Furthermore, RNF6 restoration in H9C2 cells with miR-26b upregulation improved the apoptosis rate again (Figure $6 \mathrm{~F}$ ), suggesting that miR-26b repressed cell apoptosis of $\mathrm{H}_{2} \mathrm{O}_{2}$-treated H9C2 cells by mediating RNF6 expression. In addition, CCK-8 assay was performed to reveal the cell 
A
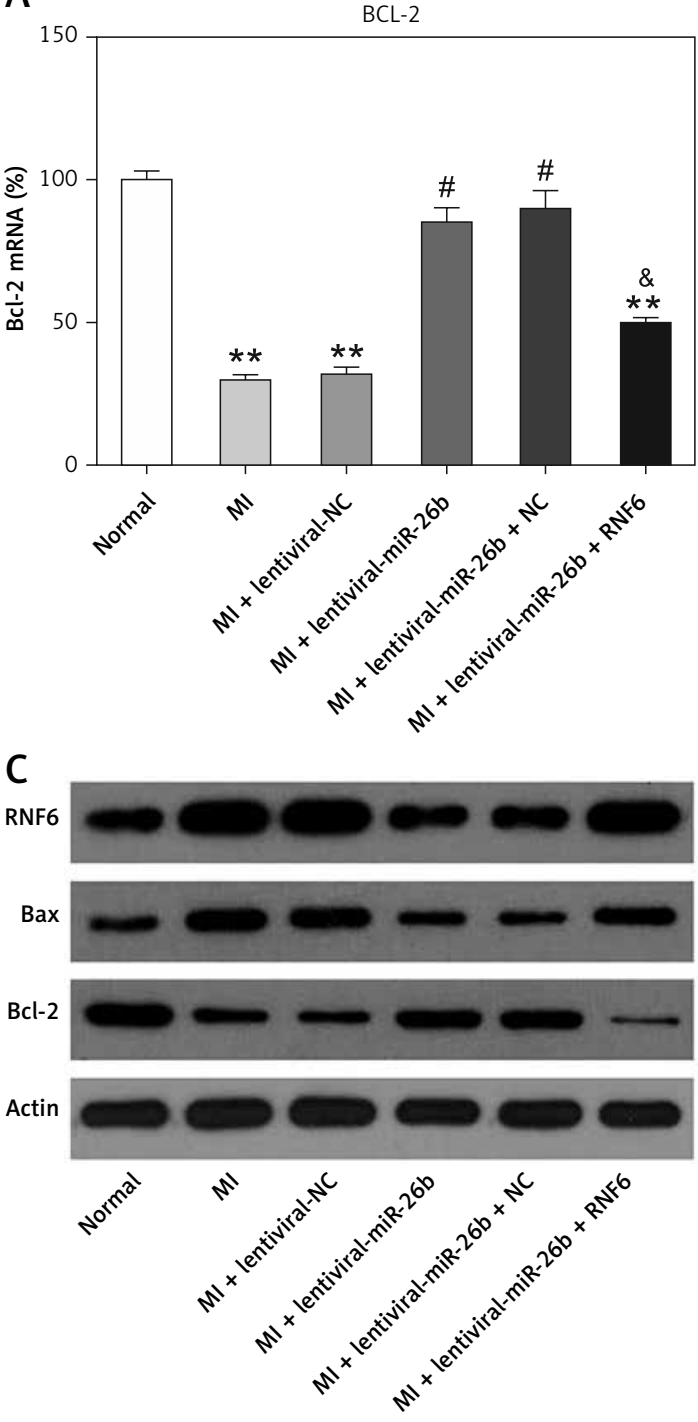

growth rate. Compared with control cells, $\mathrm{H}_{2} \mathrm{O}_{2}$ incubation led to a lower growth rate of $\mathrm{H}_{2} \mathrm{O}_{2}$-treated $\mathrm{H} 9 \mathrm{C} 2$ cells, whereas transfection with miR-26b mimic caused recovery of their proliferation rate. Notably, RNF6 overexpression obviously reduced the growth rate of $\mathrm{H}_{2} \mathrm{O}_{2}$-treated $\mathrm{H} 9 \mathrm{C} 2$ cells, which was promoted by miR-26b upregulation (Figure $6 \mathrm{G}$ ). These data suggested that miR-26b upregulation promoted survival and inhibited apoptosis of $\mathrm{H}_{2} \mathrm{O}_{2}$-treated $\mathrm{H} 9 \mathrm{C} 2$ cells by targeting RNF6.

\section{Discussion}

Myocardial remodeling (MR) is one of the main changes in cardiac structure, which leads to hemodynamic imbalance in the presence of pathological stimuli and biomechanical stress. MR involves alterations in structure, size, mass, shape, and functions. Its main causes include myocardial hypertrophy, cardiomyocyte impairment, angiogenesis, myocardial fibrosis, and inflammation. An
B

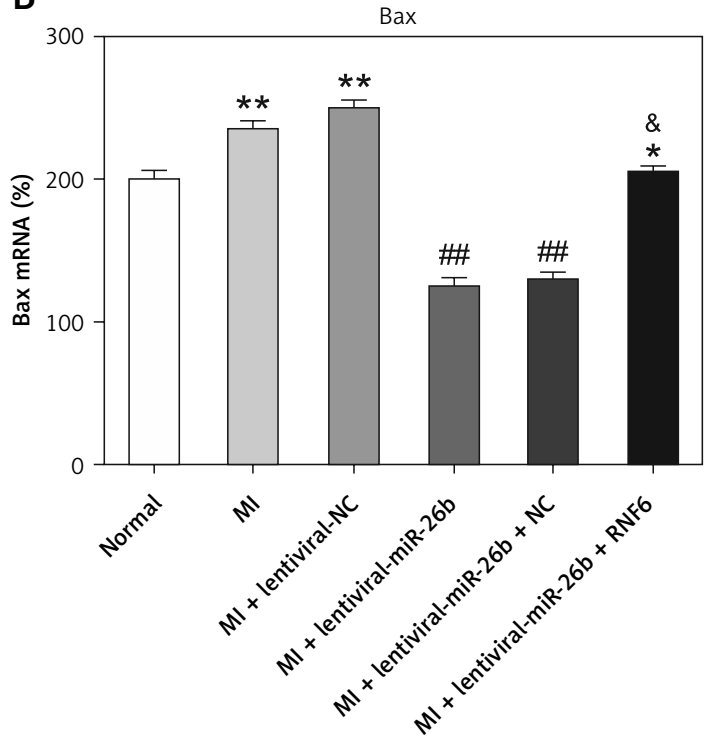

Figure 5. MicroRNA-26b (miR-26b) protects cardiomyocytes against apoptosis in $\mathrm{Ml}$ rats. The $\mathrm{MI}$ rats used were injected with lentiviral vectors expressing miR-NC, miR-26b, miR-26b+NC, and/or miR-26b+RNF6. A, B - RT-qPCR was performed to determine Bcl-2 and Bax mRNA expression levels in myocardial tissues from control and $\mathrm{MI}$ rats. $\mathrm{C}-\mathrm{WB}$ was performed to detect $\mathrm{Bcl}-2$ and Bax protein expression levels in myocardial tissues from control and $\mathrm{MI}$ rats

${ }^{* *} p<0.01$ vs. control rats, ${ }^{\#} p<0.05,{ }^{\# \#} p<0.01$ vs. MI rats, ${ }^{\&} p<0.05$ vs. MI rats injected with lentiviral vector expressing miR-26b.

obvious cardiomyocyte loss due to diverse insults and slower cardiomyocyte regeneration rate is an indicator of MR in human and animal models [32]. This study profiled miR expression in an $\mathrm{MI}$ rat model to recognize abnormally expressed cardiac miRs with latent expression in adverse CR after MI. This study revealed that the change in miR expression after $\mathrm{Ml}$ is relative to that in normal conditions. Several miRs with different expression were recognized to respond to ischemia-induced impairment, and postischemia remodeling was associated with myocardial ischemia and CR resulting in HF, such as miR-101, miR-126, miR-202, and miR-351 [33]. We observed a significant decrease in miR-26b expression level in myocardial tissues of rats after MI. miR-26 has various roles in modulating key aspects of cell growth, development, and activation. miR-26b is extremely conservative and serves as a modulator of tumor progression [34-36] and other diseases [37-39]. 
A

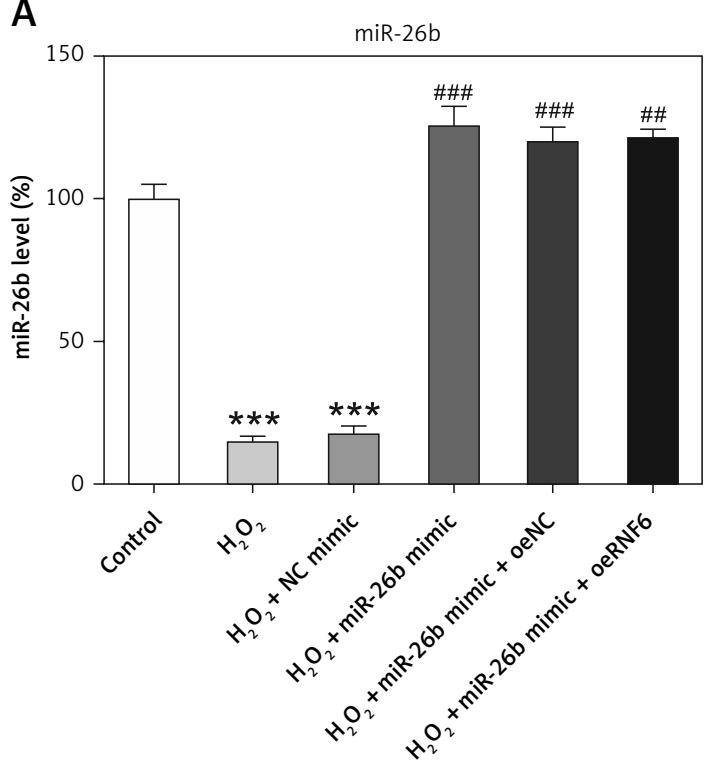

C

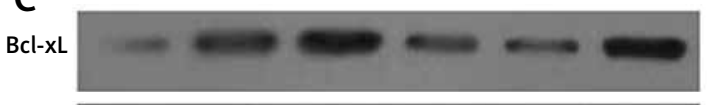

$\mathrm{ERO}$

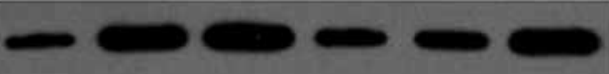

$\mathrm{Bcl}-2$

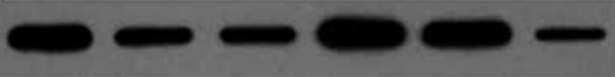

Bax

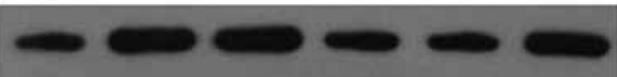

RNF6

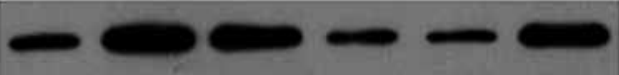

Actin
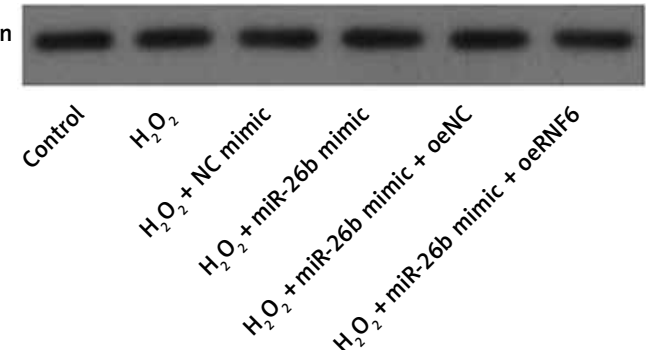

Figure 6. MicroRNA-26b (miR-26b) overexpression promoted survival and inhibited apoptosis of $\mathrm{H}_{2} \mathrm{O}_{2}$-treated $\mathrm{H} 9 \mathrm{C} 2$ cells. Cells were transfected with miR-26b mimic, NC mimic, or miR-26b+pcDNA3-NC or miR-26b+pcDNA3-RNF6 and subsequently treated with $100 \mu \mathrm{M} \mathrm{H}_{2} \mathrm{O}_{2}$ for $48 \mathrm{~h}$. A, B - RT-qPCR revealed miR-26b and RNF6 mRNA expression levels. $\mathrm{C}-\mathrm{WB}$ detected RNF6, Bcl-2, and Bax protein expression levels. D, E - RT-qPCR detected Bcl-2 and Bax mRNA expression levels

${ }^{* *} p<0.01,{ }^{* * *} p<0.001$ vs. control group. ${ }^{* \#} p<0.01$, $\# \# p<0.001$ vs. $\mathrm{H}_{2} \mathrm{O}_{2}$ treatment group, ${ }^{\& \&} p<0.01$ vs. $\mathrm{H}_{2} \mathrm{O}_{2}+$ miR-26b mimic group.
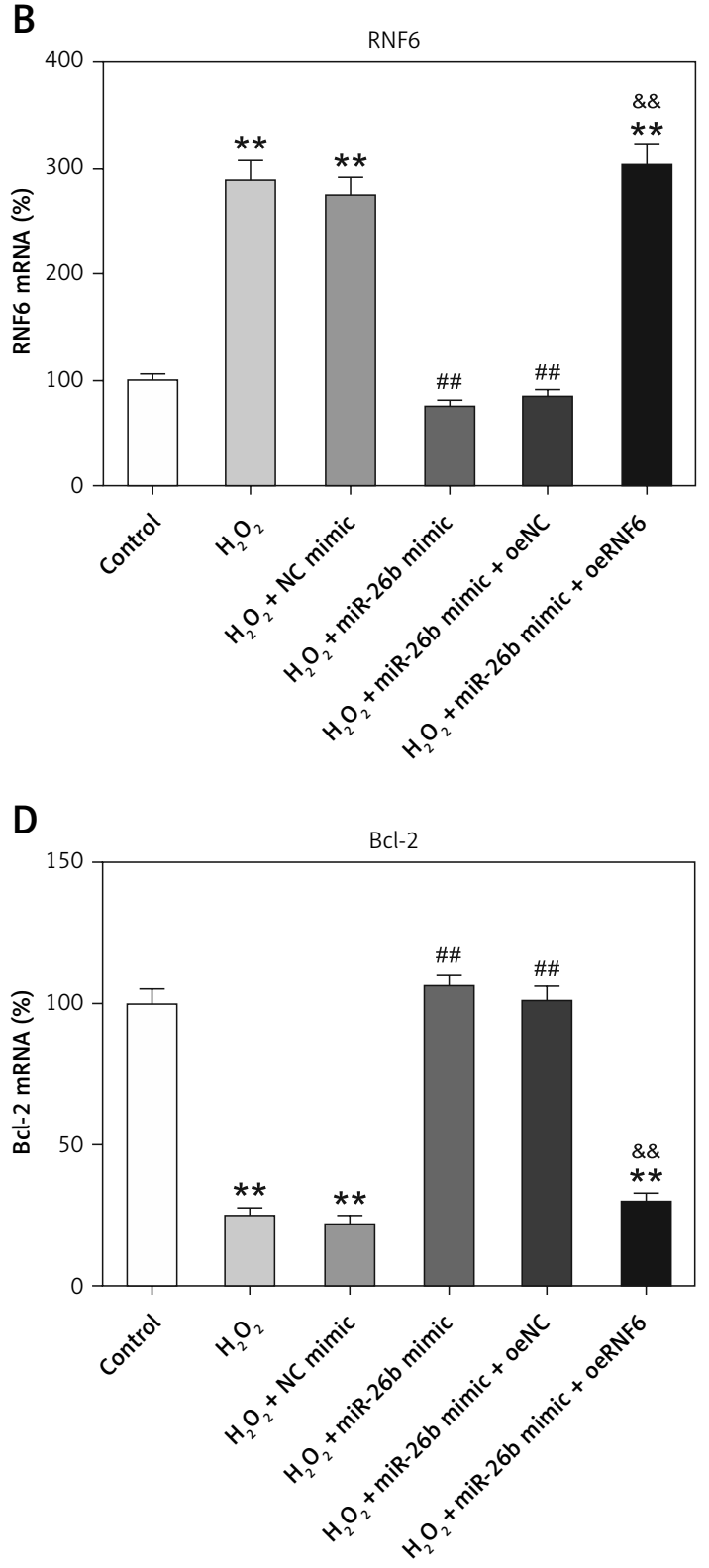

E

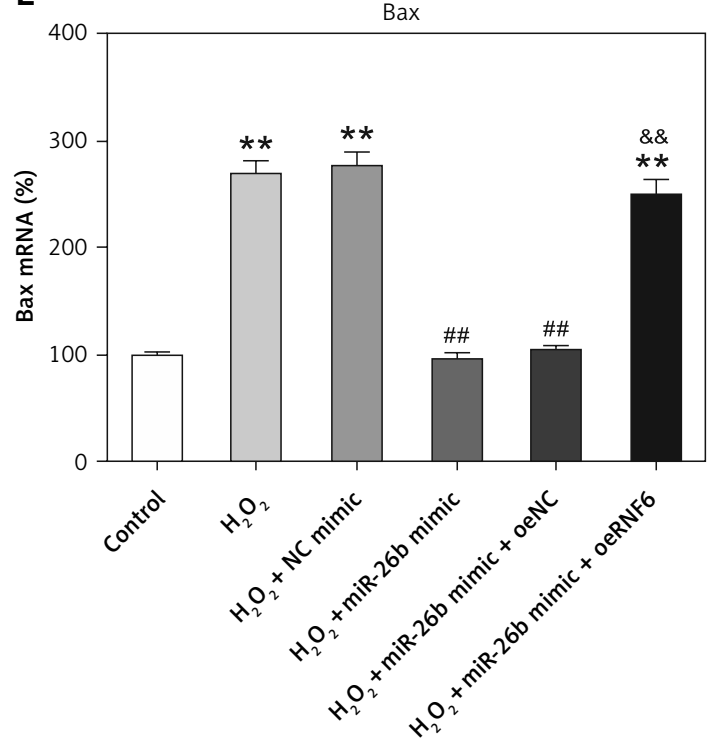


$\mathrm{F}$

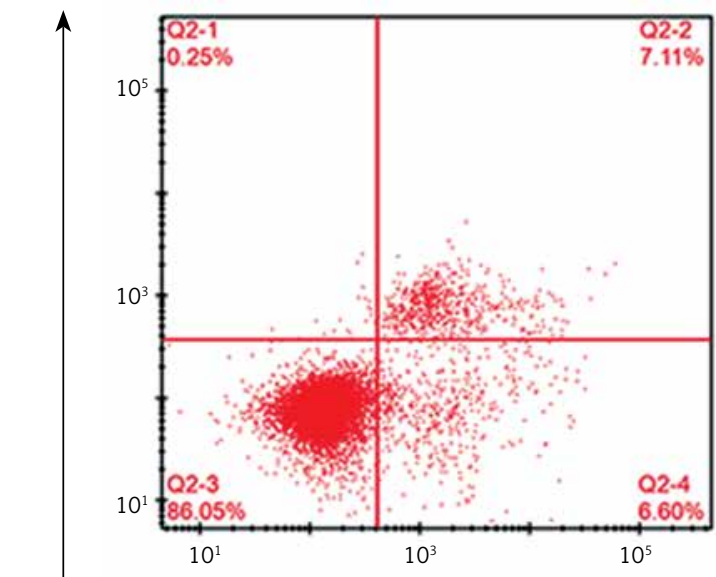

PI
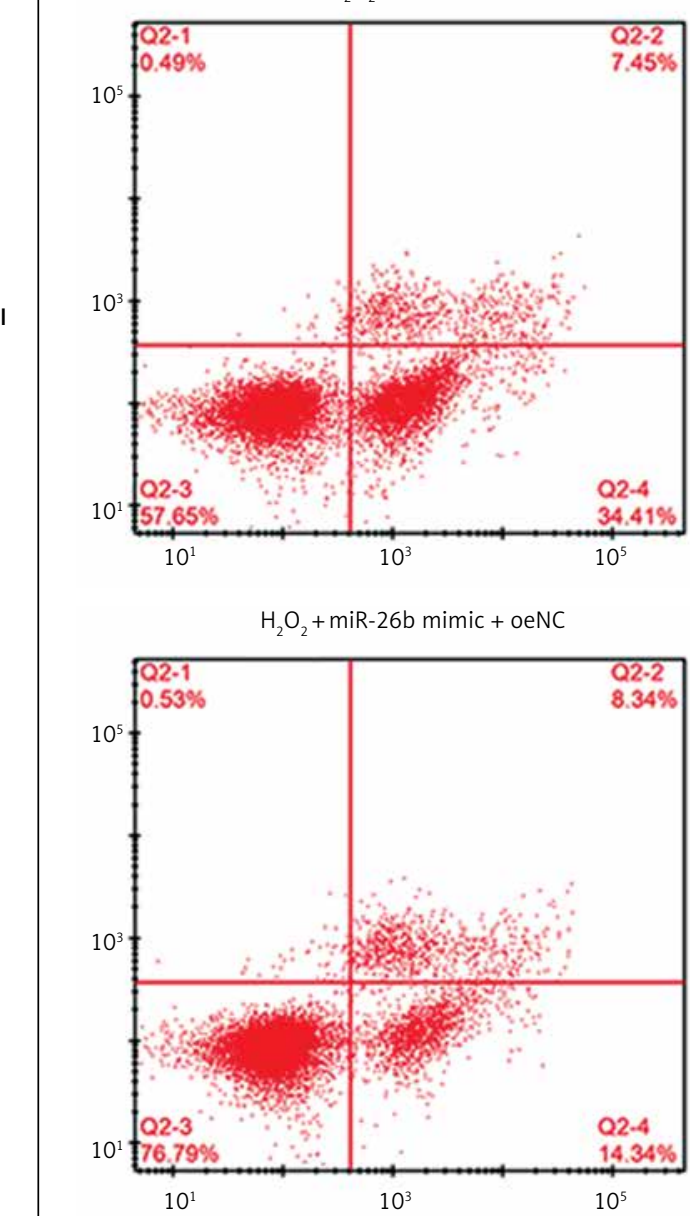

$\mathrm{H}_{2} \mathrm{O}_{2}$

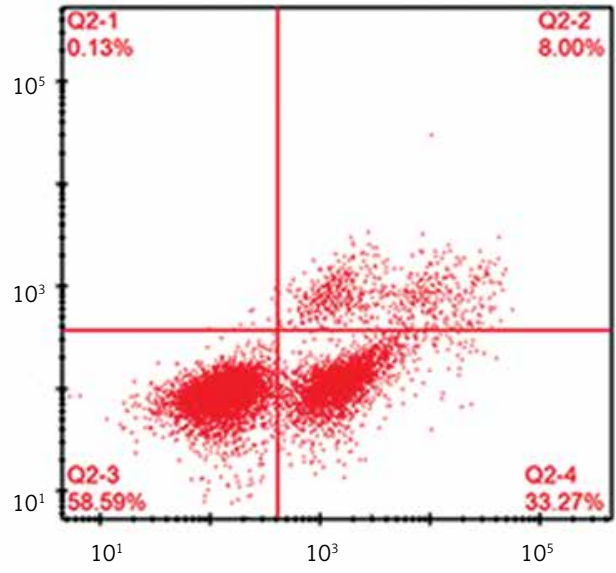

$\mathrm{H}_{2} \mathrm{O}_{2}+$ miR-26b mimic

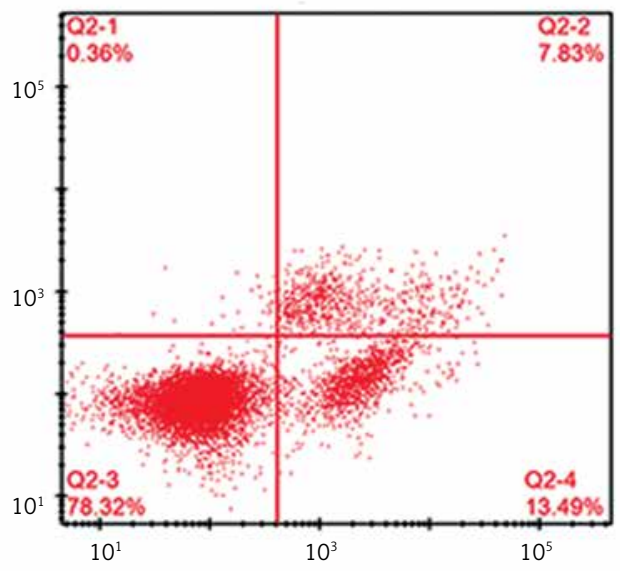

$\mathrm{H}_{2} \mathrm{O}_{2}+$ miR-26b mimic + oeRNF6

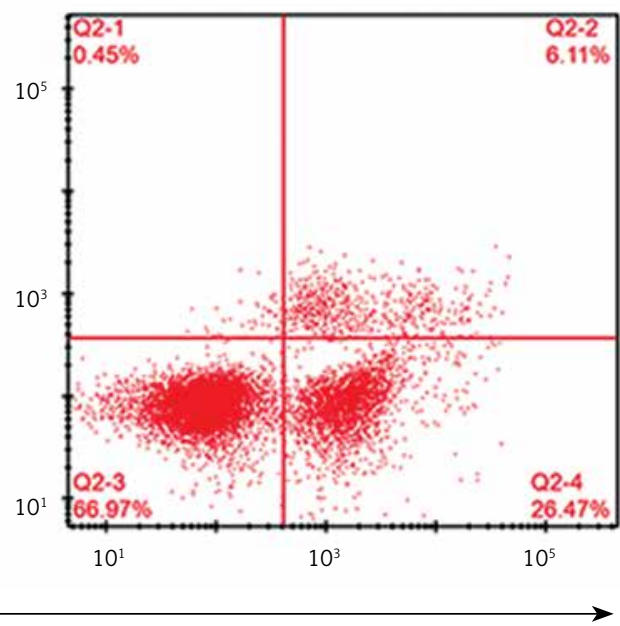

$26.47 \%$

AnnexinV-FITC

Figure 6. Cont. $\mathrm{F}$ - Effect of miR-26b on cell apoptosis was assessed by Annexin V/PI FCM

${ }^{* *} p<0.01,{ }^{* * *} p<0.001$ vs. control group. ${ }^{\# \# p}<0.01$, ${ }^{\# \# *} p<0.001$ vs. $\mathrm{H}_{2} \mathrm{O}_{2}$ treatment group, ${ }^{8 *} p<0.01$ vs. $\mathrm{H}_{2} \mathrm{O}_{2}+$ miR-26b mimic group. 
G
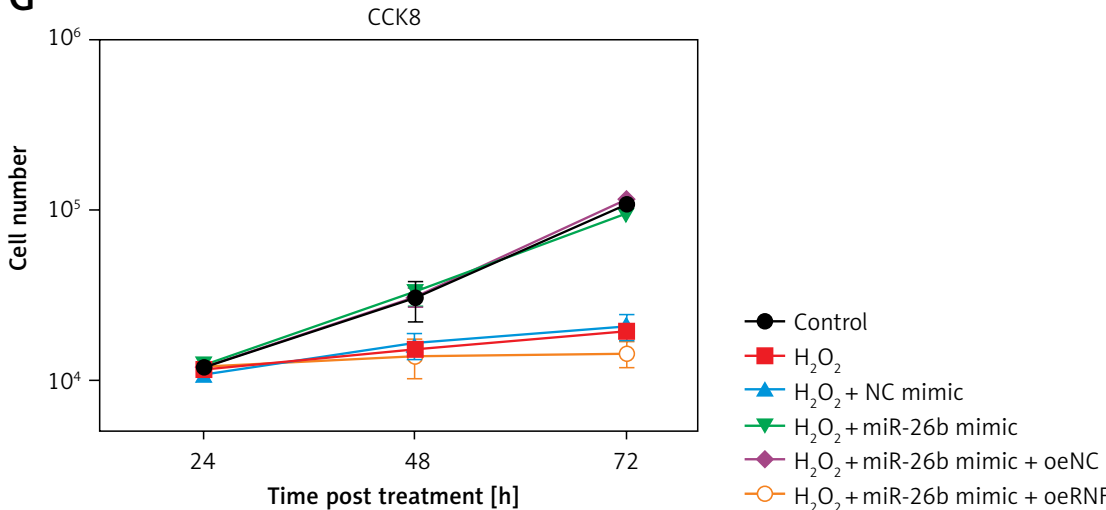

Figure 6. Cont. G - Effect of miR-26b on cell growth was assessed via CCK-8 assay

${ }^{* *} p<0.01,{ }^{* * *} p<0.001$ vs. control group. ${ }^{\# \# p}<0.01$, ${ }^{\# \#} p<0.001$ vs. $\mathrm{H}_{2} \mathrm{O}_{2}$ treatment group, ${ }^{*} \mathrm{p}<0.01$ vs. $\mathrm{H}_{2} \mathrm{O}_{2}+$ miR-26b mimic group.

Recent evidence confirms that the miR-26 family performs a vital role in cardiovascular diseases by regulating the main signaling pathways such as BMP/SMAD1 and targets associated with endothelial cell growth, angiogenesis, and LV function after MI [40]. A study reported that miR-26b was dysregulated during myocardial hypertrophy and was involved in cardiomyocyte growth and survival. miR-26b overexpression inhibited endothelin-induced hypertrophy and GATA4-related transcription as well as enhancing cell sensitivity to apoptosis-induced impairment. Moreover, miR$26 \mathrm{~b}$ acted on PLC- $\beta 1$, which then suppressed miR$26 \mathrm{~b}$ expression [21]. Zhang et al. reported that miR-26b inhibited GSK-3 $\beta$ 3'-UTR and decreased $\beta-M H C$ and ANF expression in CM in vitro, revealing the possible relevance of miR-26b to regulation of myocardial hypertrophy [41]. In the present study, we found that miR-26b upregulation suppressed adverse CR after $\mathrm{MI}$ in an $\mathrm{MI}$ rat model. miR-26b overexpression in MI maintained systolic and contractile functions in a dose-dependent manner. LVEDD, LVESD, LVEF, and LVFS are global nonintrusive measurement indicators of $C R$, which can be used for predicting clinical results after $\mathrm{MI}$ and applied to a series of cardiovascular system-based anti-remodeling treatments [42]. An absolute $20 \%$ reduction in LVEDD, $15 \%$ reduction in LVESD, and 30\% improvement in LVEF and LVFS were observed in miR-26b-upregulated cells in rats 14 days after $M I$ induction, whereas $M I$ rats revealed deterioration in $\mathrm{EF}$, suggesting analogous effects of miR-26b in clinical practice, which are particularly favorable and relevant to a notable increase in survival rate [42].

Furthermore, we inferred that the underlying mechanism of miR-26b in MI was negatively targeting RNF6 and the downstream ER $\alpha / \mathrm{Bcl}-\mathrm{xL}$ axis. RNF6 serves as a promising promoter in cancers such as hepatocellular carcinoma (HCC) and breast cancer (BC). In HCC, RNF6 knockdown suppressed HCC migratory abilities, EMT, and radioresistance [43]. In BC, an RNF6/ER $\alpha / B c l-x L$ (hereinafter abbreviated as R/E/B) axis was found to exist that facilitated cell proliferation and survival, and acting upon the R/E/B axis provided a promising treatment choice [44]. However, there is no evidence linking RNF6 to cardiovascular disease. The study showed that miR-26b acted on RNF6 mRNA and suppressed its expression. miR-26b displayed its myocardial protective activity by suppressing RNF6 expression and R/E/B signaling in $\mathrm{MI}$ rats and $\mathrm{H}_{2} \mathrm{O}_{2}$-treated $\mathrm{H} 9 \mathrm{C} 2$ cells. RNF6 restoration reversed the improved cardiac function and reduced myocardial apoptosis induced by miR-26b in vitro and in vivo. In addition, the in vitro study suggested that miR-26b inactivated $E R \alpha / B c l-x L$ signaling. The study data also suggested the role of the $\mathrm{R} / \mathrm{E} / \mathrm{B}$ axis as an apoptosis inducer in $\mathrm{H}_{2} \mathrm{O}_{2}$-treated $\mathrm{H} 9 \mathrm{C} 2$ cells, which is in contrast with the findings of a previous study in $\mathrm{BC}$ cells, possibly because of the use of different cell lines and treatment.

We found that miR-26b upregulation protected against $\mathrm{Ml}$-induced $\mathrm{CR}$ by inhibiting RNF6 expression in the $\mathrm{MI}$ rat model. Meanwhile, miR-26b disrupts the R/E/B axis, indicating that the miR26b/RNF6/ER $\alpha / B c l-x L$ axis could provide deeper knowledge on the mechanism underlying MI. Although the present data revealed promising targets for MI, further efforts are needed to validate the effectiveness of miR-26b-targeted therapies in clinical treatments. Moreover, the effects of overexpressed or silenced miR-26b in HF animal models are expected to be investigated in the future.

\section{Acknowledgments}

This work was supported by the Nanchong City University Science and Technology Strategic Cooperation Project (grant number 18SXHZ0230); 
Scientific Research Development Plan Project of North Sichuan Medical College (grant number CBY17-A-2D12); Nanchong Key Laboratory of Individualized Drug Therapy (grant number NCKL201711) and Sichuan Provincial Education Department Fund (grant number 18ZB0221).

\section{Conflict of interests}

The authors declare no conflict of interest.

\section{References}

1. Cowie M, Mosterd A, Wood D, Deckers J, Poole-Wilson P, Sutton G, et al. The epidemiology of heart failure. Eur Heart J 1997; 18: 208-225.

2. Mozaffarian D, Benjamin EJ, Go AS, Arnett DK, Blaha MJ, Cushman $M$, et al. Executive summary: heart disease and stroke statistics - 2016 update: a report from the American Heart Association. Circulation 2016; 133: 447-454.

3. Piątek Ł, Wilczek K, Kurzawski J, Gierlotka M, Gąsior M, Poloński L, et al. Gender-related disparities in the treatment and outcomes in patients with non-ST-segment elevation myocardial infarction: results from the Polish Registry of Acute Coronary Syndromes (PL-ACS) in the years 2012-2014. Arch Med Sci 2020; 16: 781-788.

4. Jonas S, Izaurralde E. Towards a molecular understanding of microRNA-mediated gene silencing. Nat Rev Genet 2015; 16: 421-433.

5. Rigoutsos I. New tricks for animal microRNAS: targeting of amino acid coding regions at conserved and nonconserved sites. Cancer Res 2009; 69: 3245-3248.

6. Werfel S, Leierseder S, Ruprecht B, Kuster B, Engelhardt $S$. Preferential microRNA targeting revealed by in vivo competitive binding and differential Argonaute immunoprecipitation. Nucleic Acids Res 2017; 45: 1021810228.

7. Mendell JT, Olson EN. MicroRNAs in stress signaling and human disease. Cell 2012; 148: 1172-1187.

8. Hata A. Functions of microRNAs in cardiovascular biology and disease. Annu Rev Physiol 2013; 75: 69-93.

9. Van Rooij E, Sutherland LB, Qi X, Richardson JA, Hill J, Olson EN. Control of stress-dependent cardiac growth and gene expression by a microRNA. Science 2007; 316 : 575-579.

10. Care A, Catalucci D, Felicetti F, Bonci D, Addario A Gallo P, et al. MicroRNA-133 controls cardiac hypertrophy. Nat Med 2007; 13: 613-618.

11. Ucar A, Gupta SK, Fiedler J, Erikci E, Kardasinski M, Batkai S, et al. The miRNA-212/132 family regulates both cardiac hypertrophy and cardiomyocyte autophagy. Nat Commun 2012; 3: 1078.

12. Duisters RF, Tijsen AJ, Schroen B, Leenders JJ, Lentink $\mathrm{V}$, van der Made I, et al. miR-133 and miR-30 regulate connective tissue growth factor: implications for a role of microRNAs in myocardial matrix remodeling. Circ Res 2009; 104: 170-178.

13. Matkovich SJ, Wang W, Tu Y, Eschenbacher WH, Dorn LE, Condorelli G, et al. MicroRNA-133a protects against myocardial fibrosis and modulates electrical repolarization without affecting hypertrophy in pressure-overloaded adult hearts. Circ Res 2010; 106: 166-175.

14. Van Rooij E, Sutherland LB, Thatcher JE, DiMaio JM, Naseem RH, Marshall WS, et al. Dysregulation of microRNAs after myocardial infarction reveals a role of miR-29 in cardiac fibrosis. Proc Natl Acad Sci USA 2008; 105: 13027-13032.

15. Thum T, Gross C, Fiedler J, Fischer T, Kissler S, Bussen M, et al. MicroRNA-21 contributes to myocardial disease by stimulating MAP kinase signalling in fibroblasts. Nature 2008; 456: 980-984.

16. Ramanujam D, Sassi Y, Laggerbauer B, Engelhardt S. Viral vector-based targeting of miR-21 in cardiac nonmyocyte cells reduces pathologic remodeling of the heart. Mol Ther 2016; 24: 1939-1948.

17. Yao L, Lv X, Wang X. MicroRNA 26a inhibits HMGB1 expression and attenuates cardiac ischemia-reperfusion injury. J J Pharmacol Sci 2016; 131: 6-12.

18. Liu Y, Wang Z, Xiao W. MicroRNA-26a protects against cardiac hypertrophy via inhibiting GATA4 in rat model and cultured cardiomyocytes. Mol Med Rep 2016; 14: 2860-2866.

19. Kong B, Qin Z, Ye Z, Yang X, Li L and Su Q. microRNA 26 a $5 p$ affects myocardial injury induced by coronary microembolization by modulating HMGA1. J Cell Biochem 2019; 120: 10756-10766.

20. Tang C-M, Zhang M, Huang L, Hu Z-q, Zhu J-N, Xiao Z, et al. CircRNA_000203 enhances the expression of fibrosis-associated genes by derepressing targets of miR26b-5p, Col1a2 and CTGF, in cardiac fibroblasts. Sci Rep 2017; 7: 1-9.

21. Han M, Yang Z, Sayed D, He M, Gao S, Lin L, et al. GATA4 expression is primarily regulated via a miR-26b-dependent post-transcriptional mechanism during cardiac hypertrophy. Cardiovasc Res 2012; 93: 645-654.

22. Wang X, Li C, Dai Q. Down-regulation of microRNA-26b rescued hypoxia-induced apoptosis in cultured neonatal rat cardiac myocytes by regulating PTEN. Int I Clin Exp Med 2015; 8: 4073-4079.

23. Ge Z-W, Zhu X-L, Wang B-C, Hu J-L, Sun J-J, Wang S, et al. MicroRNA-26b relieves inflammatory response and myocardial remodeling of mice with myocardial infarction by suppression of MAPK pathway through binding to PTGS2. Int J Cardiol 2019; 280: 152-159.

24. Huang ZM, Ge HF, Yang CC, Cai Y, Chen Z, Tian WZ, et al. MicroRNA-26a-5p inhibits breast cancer cell growth by suppressing RNF6 expression. Kaohsiung J Med Sci 2019; 35: 467-473.

25. Martinez EC, Vu D-T, Wang J, Lilyanna S, Ling L H, Gan $\mathrm{SU}$, et al. Grafts enriched with subamnion-cord-lining mesenchymal stem cell angiogenic spheroids induce post-ischemic myocardial revascularization and preserve cardiac function in failing rat hearts. Stem Cells Dev 2013; 22: 3087-3099.

26. Lilyanna S, Peh MT, Liew OW, Wang P, Moore PK, Richards AM, et al. GYY4137 attenuates remodeling, preserves cardiac function and modulates the natriuretic peptide response to ischemia. J Mol Cell Cardiol 2015; 87: 27-37.

27. Karolina DS, Tavintharan S, Armugam A, Sepramaniam $\mathrm{S}$, Pek SLT, Wong M T, et al. Circulating miRNA profiles in patients with metabolic syndrome. The J Clin Endocrinol Metab 2012; 97: E2271-E2276.

28. Xiang L, Wang M, You T, Jiao Y, Chen J, Xu W. Prognostic value of ventricular wall motion score and global registry of acute coronary events score in patients with acute myocardial infarction. Am J Med Sci 2017; 354: 27-32.

29. Xu X, Wang J, Han K, Li S, Xu F, Yang Y. Antimalarial drug mefloquine inhibits nuclear factor kappa B signaling and induces apoptosis in colorectal cancer cells. Cancer Sci 2018; 109: 1220-1229.

30. Zhang S, Song X. Long non-coding RNA SNHG1 promotes cell proliferation and invasion of hepatocellular 
carcinoma by acting as a molecular sponge to modulate miR-195. Arch Med Sci 2019; 16: 386-394.

31. Qiu L, Chen J, Lin J, Wo D, Chu J, Peng J. Baicalin alleviates $\mathrm{H}_{2} \mathrm{O}_{2}$-induced injury of $\mathrm{H} 9 \mathrm{c} 2$ cardiomyocytes through suppression of the Wnt/betacatenin signaling pathway. Mol Med Rep 2017; 16: 9251-9255.

32. Dash R, Kadambi VJ, Schmidt AG, Tepe NM, Biniakiewicz D, Gerst MJ, et al. Interactions between phospholamban and $\beta$-adrenergic drive may lead to cardiomyopathy and early mortality. Circulation 2001; 103: 889-896.

33. Da Silva W, dos Santos RAS, Moraes KC. Mir-351-5p contributes to the establishment of a pro-inflammatory environment in the H9c2 cell line by repressing PTEN expression. Mol Cell Biochem 2016; 411: 363-371.

34. Palumbo T, Faucz FR, Azevedo M, Xekouki P, Iliopoulos $D$, Stratakis CA. Functional screen analysis reveals miR$26 \mathrm{~b}$ and miR-128 as central regulators of pituitary somatomammotrophic tumor growth through activation of the PTEN-AKT pathway. Oncogene 2013; 32: 1651.

35. Li Y, Sun Z, Liu B, Shan Y, Zhao L, Jia L. Tumor-suppressive miR-26a and miR-26b inhibit cell aggressiveness by regulating FUT4 in colorectal cancer. Cell Death Dis 2017; 8: e2892-e2892.

36. Tsai M-M, Huang H-W, Wang C-S, Lee K-F, Tsai C-Y, Lu P-H, et al. MicroRNA-26b inhibits tumor metastasis by targeting the KPNA2/c-jun pathway in human gastric cancer. Oncotarget 2016; 7: 39511.

37. Absalon S, Kochanek DM, Raghavan V, Krichevsky AM MiR-26b, upregulated in Alzheimer's disease, activates cell cycle entry, tau-phosphorylation, and apoptosis in postmitotic neurons. J Neurosci 2013; 33: 14645-14659.

38. Lin F, Li R, Xiang Pan Z, Zhou B, Bing Yu D, Guang Wang $X$, et al. miR-26b promotes granulosa cell apoptosis by targeting ATM during follicular atresia in porcine ovary. PLoS One 2012; 7: e38640.

39. Wang J, Zhang Y, Zhang W, Jin Y, Dai J. Association of perfluorooctanoic acid with HDL cholesterol and circulating miR-26b and miR-199-3p in workers of a fluorochemical plant and nearby residents. Environ Sci Technol 2012; 46: 9274-9281.

40. Icli B, Dorbala P, Feinberg MW. An emerging role for the miR-26 family in cardiovascular disease. Trends Cardiovasc Med 2014; 24: 241-248.

41. Zhang Z-h, Li J, Liu B-r, Luo C-f, Dong Q, Zhao L-n, et al. MicroRNA-26 was decreased in rat cardiac hypertrophy model and may be a promising therapeutic target. J Cardiovasc Pharmacol 2013; 62: 312-319.

42. Sekaran NK, Crowley AL, de Souza FR, Resende ES, Rao SV. The role for cardiovascular remodeling in cardiovascular outcomes. Curr Atheroscler Rep 2017; 19: 23.

43. Cai J, Xiong Q, Jiang X, Zhou S, Liu T. RNF6 facilitates metastasis and radioresistance in hepatocellular carcinoma through ubiquitination of FoxA1. Exp Cell Res 2019; 374: 152-161.

44. Zeng Y, Xu X, Wang S, Zhang Z, Liu Y, Han K, et al. Ring finger protein 6 promotes breast cancer cell proliferation by stabilizing estrogen receptor alpha. Oncotarget 2017; 8: 20103-20112. 